\title{
Measurements of Heat Capacity and Enthalpy of Phase Change Materials by Adiabatic Scanning Calorimetry
}

\author{
Patricia Losada-Pérez • Chandra Shekhar Pati Tripathi • \\ Jan Leys • George Cordoyiannis • Christ Glorieux • \\ Jan Thoen
}

Received: 26 August 2010 / Accepted: 23 March 2011 / Published online: 20 April 2011 (C) Springer Science+Business Media, LLC 2011

\begin{abstract}
Phase change materials (PCMs) are substances exhibiting phase transitions with large latent heats that can be used as thermal storage materials with a large energy storage capacity in a relatively narrow temperature range. In many practical applications the solid-liquid phase change is used. For applications accurate knowledge of different thermal parameters has to be available. In particular, the temperature dependence of the enthalpy around the phase transition has to be known with good accuracy. Usually, the phase transitions of PCMs are investigated with differential scanning calorimetry (DSC) at fast dynamic scanning rates resulting in the effective heat capacity from which the (total) heat of transition can be determined. Here we present adiabatic scanning calorimetry (ASC) as an alternative approach to arrive simultaneously at the equilibrium enthalpy curve and at the heat capacity. The applicability of ASC is illustrated with measurements on paraffin-based PCMs and on a salt hydrate PCM.
\end{abstract}

Keywords Adiabatic scanning calorimetry $\cdot$ Enthalpy $\cdot$ Heat capacity · Latent heat · Phase change materials

\footnotetext{
P. Losada-Pérez · C. S. P. Tripathi · J. Leys $(\varangle) \cdot$ G. Cordoyiannis · C. Glorieux · J. Thoen Laboratorium voor Akoestiek en Thermische Fysica, Departement Natuurkunde en Sterrenkunde, Katholieke Universiteit Leuven, Celestijnenlaan 200D, 3001 Leuven, Belgium e-mail: jan.leys@fys.kuleuven.be

J. Thoen

e-mail: jan.thoen@fys.kuleuven.be

Present Address:

G. Cordoyiannis

Condensed Matter Physics Departement, Jožef Stefan Institute, Jamova 39, 1000 Ljubljana, Slovenia
} 


\section{Introduction}

Phase change materials (PCMs) are substances exhibiting phase transitions with large latent heats and can be used as thermal storage materials with a large energy storage capacity in a relatively narrow temperature range [1-3]. In principle latent heat storage can be achieved through a solid-solid, solid-liquid, solid-gas, or liquid-gas phase change. In practical applications where temperature control is important, the solid-liquid phase change is mostly used. In nowadays applications mainly two types of PCMs are in use. A first category includes organic materials, mainly paraffins and to some extent fatty acids. The second large category includes the inorganic salt hydrates. Recently, also eutectic mixture combinations of organic and non-organic compounds are being considered. The choice of material depends not only on the intrinsic properties of the PCM but also on the practical application envisioned. A large number of PCMs are available in the temperature range from well below $0{ }^{\circ} \mathrm{C}$ to $200^{\circ} \mathrm{C}$.

For proper design of application devices an accurate knowledge of different thermal parameters has to be available. In particular, the temperature dependence of the enthalpy around the phase transition has to be known with good accuracy. Usually, the phase transitions of PCMs are investigated with differential scanning calorimetry (DSC). In DSC a reference sample is made to increase (or decrease) its temperature at a constant rate and the PCM sample is forced to follow this rate by changing the power delivered to it. This allows one to extract the (effective) heat capacity as a function of temperature. The transition heat is then determined by integrating the heat capacity curve. Moreover, DSC uses (for sufficient resolution) fast scanning rates $\left(1 \mathrm{~K} \cdot \mathrm{min}^{-1}\right.$ to $10 \mathrm{~K} \cdot \min ^{-1}$ ), quite often resulting in (apparent) overheating and undercooling effects. Efforts to (partly) overcome these problems for latent heat measurements of PCMs have resulted in running a DSC in an isothermal step mode and/or by applying a $T$-history method [4].

In this paper we present adiabatic scanning calorimetry (ASC) as an interesting complementary tool to measure simultaneously the temperature dependence of the enthalpy as well as of the heat capacity near the phase transitions in PCMs. ASC has been extensively used to discriminate between first-order (exhibiting a discontinuous step in the enthalpy) and second-order (with a continuous temperature dependence of the enthalpy) phase transitions and to detect heat-capacity anomalies near critical points in several types of soft matter systems, such as, e.g., liquid crystals and critical mixtures [5-8]. With ASC the problems with superheating or supercooling can in many cases be avoided and true equilibrium data can be obtained by using very slow rates as slow as 2 to 3 orders of magnitude slower than in DSC. After a description of the ASC technique, we present results for the temperature dependence of the enthalpy and of the (effective) heat capacity of two paraffin-based PCMs and of one pure salt hydrate. In addition, also results for a paraffin-based powder PCM are given.

\section{Experimental Method}

The so-called ASC technique was introduced around 1980 [5] and extensively used for the study of many different types of phase transitions, in particular in liquid 
mixtures and liquid crystals. An extensive description of the technique and major results can be found in recent overviews $[7,8]$. Here we will only give a description of the two principal modes of operation of an ASC and some key features of the data analysis.

\subsection{Principal Modes of Operation of an ASC}

Since the beginning of the twentieth century, several different calorimetric techniques with varying degrees of accuracy and precision have been developed. Traditionally, heat-capacity measurements are carried out by means of the adiabatic heat pulse method, where a known amount of heat, $\Delta Q$, is (usually electrically) applied to the sample and the corresponding temperature rise, $\Delta T$, is measured. The heat capacity (at constant pressure) of a sample at a given temperature is then obtained from

$$
C_{p}=\frac{\Delta Q}{\Delta T}
$$

In this way one looks at the derivative of the enthalpy $H(T)$ curve and no information can be obtained on enthalpy discontinuities or latent heats (and thus on the order of a given phase transition). Rewriting Eq. 1 in the following way:

$$
C_{p}=\frac{\mathrm{d} Q}{\mathrm{~d} T}=\frac{\mathrm{d} Q / \mathrm{d} t}{\mathrm{~d} T / \mathrm{d} t}=\frac{P}{\dot{T}}
$$

(with $t$ time and $P$ power), shows the possibility of operating in dynamic modes. By keeping $P$ or $\dot{T}$ constant, while increasing or decreasing the temperature of the sample ( $P$ and $\dot{T}$ positive or negative), four practical modes of operation are obtained. These modes require different settings for the (adiabatic) thermal environment (thermal shields) of the sample. The most interesting modes (and for enthalpy measurements where latent heats are present, the only feasable ones) are the ones with constant heating or cooling power $P$. Since in the PCM phase transitions substantial latent heats are present, we will only give a general description of the constant power modes. Detailed information on all modes can be found elsewhere [8].

In the heating mode with constant (electrically applied) power $P^{\mathrm{e}}$ to the sample (cell), in order to maintain the adiabatic conditions, one has to arrange for negligibly small leaking power $P^{1}$ to the environment, measure $P^{\mathrm{e}}$ and carefully follow the evolution of the sample temperature $T(t)$ with time $t$. Because the heating rate is inversely proportional to $C_{p}$, the increase of $C_{p}$ at a second-order phase transition will result in a decrease of the rate and facilitate thermodynamic equilibrium and servo-control of adiabatic conditions. At first-order transitions, in principle, the rate is zero at the transition for a time interval given by

$$
\Delta t=t_{\mathrm{f}}-t_{\mathrm{i}}=\frac{L}{P^{\mathrm{e}}}
$$


where $L$ is the latent heat of the transition, and $t_{\mathrm{i}}$ and $t_{\mathrm{f}}$ are the times during the scan at which the transition is reached and left. The direct experimental result $T(t)$ gives the enthalpy as a function of temperature by

$$
H=H\left(T_{0}\right)+P^{\mathrm{e}}\left(t-t_{0}\right)
$$

with $T_{0}$ the starting temperature of the scanning run at the time $t_{0}$. Implementing a cooling run with constant (negative) power is less obvious and has to be realized by imposing a constant leaking power between the sample (cell) and its isothermal environment. This can be done by imposing a constant temperature difference between the cell and the isothermal environment. These conditions have to be verified and usually involve calibration (certainly for scans over large temperature ranges) to arrive at absolute values for the heat capacity or enthalpy. This type of cooling run (with negative power and negative rate) is very similar to the constant power heating mode and also easily allows one to deal with first-order transitions. Although an ASC is normally optimized for scanning, it can easily be operated as a normal heat pulse step calorimeter as well. This can be very practical for calibration purposes and verification of absolute heat-capacity values.

\subsection{Implementation of the ASC Concept}

Figure 1 gives a schematic diagram of a four-stage ASC that can operate between room temperature and about $470 \mathrm{~K}$. The centrally located cylindrical sample cell for liquids is surrounded by three concentric (copper) thermal shields. Each of the stages (1 to 4) has its own thermometer $\left(\mathrm{Th}_{i}\right)$ and its own electrical (e.g., constantan) heating wires. On stages 1 to 3 the heating wires are evenly distributed and wound in grooves and thermally anchored with a good thermal conductive and electrically insulating epoxy. Stage 4 of this calorimeter is composed of a hot air oven and the outer thermal and vacuum shield of the actual calorimeter with three internal stages. The temperature of the oven is measured and controlled by means of the thermistor $\mathrm{Th}_{4}$ and computer regulated power delivery to the heater of the oven. The stages are in very poor thermal contact, and the space between them is vacuum pumped. The sample cell is suspended by thin nylon threads inside stage 2 . To minimize further thermal transfer between stages, all electric connecting wires are, on passing from one stage to another, several thermal diffusion lengths long (for temperature variations at relevant time scales), and neatly coiled not to touch the wall of either stage. These wires are also thermally anchored at each stage. Different sizes of sample cells can be suspended in the calorimeter. For the PCM measurements we used cells with a volume of $5 \mathrm{~cm}^{3}$ to $11 \mathrm{~cm}^{3}$. For liquid samples it is also possible to stir the samples inside the cell. Stirring in the horizontally mounted cylindrical cells is achieved by means of a metal ball that can roll back and forth inside the cell by changing periodically the inclination of the plate supporting the calorimeter. 
Fig. 1 Schematic diagram of a four-stage ASC with typical modern measurement and PC controlled instrumentation. (1) sample in sample holder with stirring ball, thermistor $\mathrm{Th}_{1}$, and heater (not shown); (2) shield with thermistor $\mathrm{Th}_{2}$, platinum reference thermometer $\mathrm{Pt}_{2}$, and heater (not shown); (3) shield with platinum thermometer $\mathrm{Pt}_{3}$ and heater (not shown); and (4) external vacuum tight shield in hot air oven with thermistor $\mathrm{Th}_{4}$ and heater. K-2010 (7.5 digit multiplexer) and HP-34401 (6.5 digits) are multimeters. There are two HP-6181B power sources and one self-made one

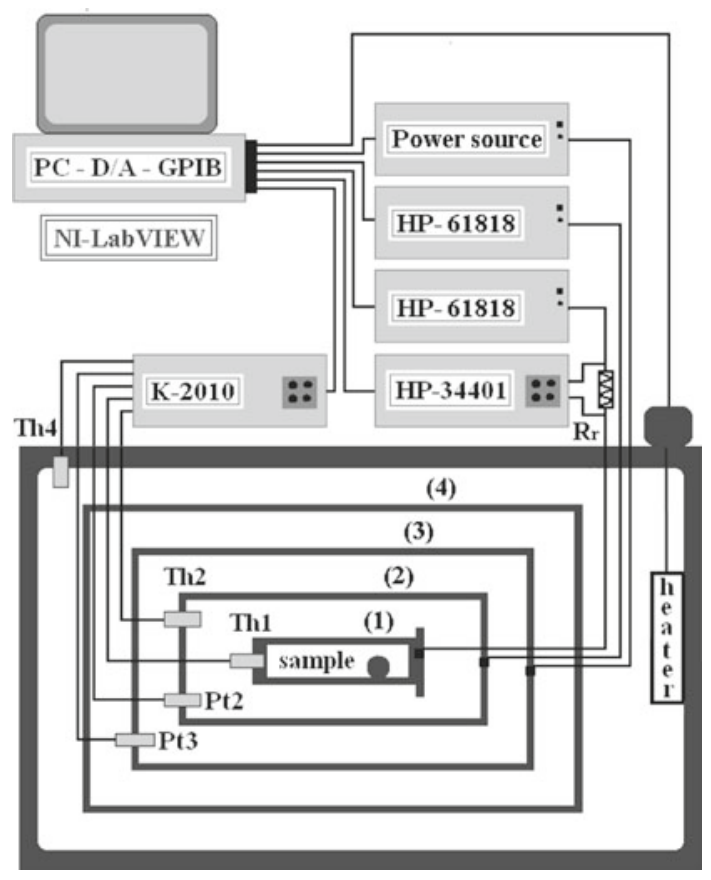

\subsection{Analysis of the Direct Experimental Data}

The basic data measured very frequently as a function of time (typically every $3 \mathrm{~s}$ to $5 \mathrm{~s}$ ) during a heating run in an ASC are the temperature of the sample and the holder as well as the (constant) power. These results are graphically displayed in the two central boxes of Fig. 2 for a weakly first-order transition. After a long temperature stabilization time of stage 2 (shield around the sample cell) with zero power to the cell (stage 1), the cell attains the same temperature (within a few tenths of a mK). Then the power to the sample cell is switched on, at $t_{0}$, to a chosen value depending on the desired overall scanning rate. Depending on the temperature range to be covered, a typical run can take several days or weeks (for very slow scans). The temperature is measured with $\mu \mathrm{K}$ resolution, and the extremely large number of $T(t)$ data allows averaging (if desired) and determination of local derivatives resulting in nearly as many $\dot{T}$ values as $T(t)$ data points by using several (consecutive) data points in a moving time derivative (adding one data point at one end and leaving out one at the other end). A simple division of $P$ by $\dot{T}$ at a given $T(t)$ results immediately in a $C_{p}(T)$ value at that temperature. These $C_{p}(T)$ values are total heat capacities for the sample and the sample holder together. Proper calibration of the (only weakly $T$ dependent) heat capacity of the empty cell and knowing the total amount of sample allows one to calculate the specific heat capacity of the sample. In the case of a first-order transition, the transition is reached at time $t_{\mathrm{i}}$. From that moment until its end at $t_{\mathrm{f}}$ the temperature remains constant over the time interval given by Eq. 3. According to Eq. 4, the direct combination of $t(T)$ and $P(t)$ data immediately results in the enthalpy as a function 
Fig. 2 Analysis of the directly obtained $T(t)$ and $P(t)$ data in a constant power heating run for a weakly first-order transition to arrive at results for the specific heat capacity $C_{p}(T)$ and the enthalpy $H(T) . T_{0}$ is the starting temperature at time $t_{0} . t_{\mathrm{i}}$ and $t_{\mathrm{f}}$ are the beginning and the end of a first-order transition at $T_{\text {tr }}$

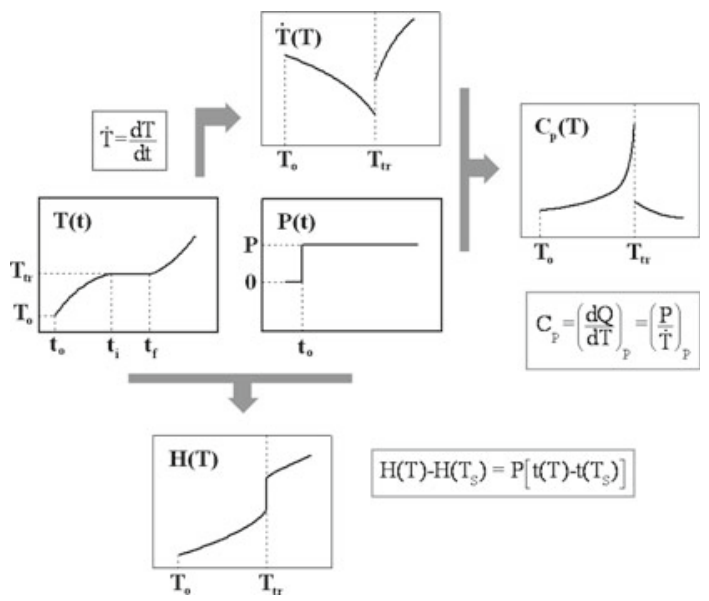

of temperature, including the latent heat discontinuity for a first-order transition as displayed in Fig. 2. The fact that in ASC the most direct result is $H(T)$ makes it a unique tool to determine the order of a phase transition. In Fig. 2, at the first-order transition, $T$ versus $t$ is depicted perfectly horizontal and $H$ versus $T$ perfectly vertical. This is, however, an idealized situation for a perfectly pure one-component sample. For real systems, even with minute amounts of impurities, as well as for two or more component mixtures, one observes a two-phase region. For single components as well as for eutectic mixtures, the two-phase region can be very small depending on the unknown (and unavoidable) small amounts of impurities. For very pure one-component systems, this two-phase region is typically a couple of tens of $\mathrm{mK}$, and depends on the impurity type and level and on the latent heat $[9,10]$.

\section{Materials}

Adiabatic scanning calorimetry (ASC) measurements were carried out for three $n$-paraffin- and waxes-based PCM materials. These materials were purchased from Rubitherm GmbH, Germany. The company names of these materials are Rubitherm RT 27, Rubitherm RT 42, and Rubitherm PX 42. The first material has a quoted melting range of $25^{\circ} \mathrm{C}$ to $28^{\circ} \mathrm{C}$ (typically being $27^{\circ} \mathrm{C}$ ) and a congealing range of $28^{\circ} \mathrm{C}$ to $25^{\circ} \mathrm{C}$ (typically being $27^{\circ} \mathrm{C}$ ) with a quoted heat storage capacity of $184 \mathrm{~kJ} \cdot \mathrm{kg}^{-1}$ (temperature range not quoted). The second one has a quoted melting range of $38^{\circ} \mathrm{C}$ to $43^{\circ} \mathrm{C}$ (typically being $41^{\circ} \mathrm{C}$ ) and a congealing range of $43^{\circ} \mathrm{C}$ to $37^{\circ} \mathrm{C}$ (typically being $42^{\circ} \mathrm{C}$ ) and a quoted heat storage capacity of $174 \mathrm{~kJ} \cdot \mathrm{kg}^{-1}$ (between $35^{\circ} \mathrm{C}$ and $50^{\circ} \mathrm{C}$ ). Rubitherm PX 42 is a heat storage powder in which a phase change material is contained within a secondary supporting structure. In this case the supporting structure is a hydrophilic silica powder. The quoted melting range is $38^{\circ} \mathrm{C}$ to $43^{\circ} \mathrm{C}$ (typically being $41^{\circ} \mathrm{C}$ ). The average particle size is $250 \mu \mathrm{m}$ and the quoted heat storage capacity is $114 \mathrm{~kJ} \cdot \mathrm{kg}^{-1}$ (temperature range $35^{\circ} \mathrm{C}$ to $50^{\circ} \mathrm{C}$ ). RT 27 was measured in a $5 \mathrm{~cm}^{3}$ thin-walled cylindrical cell with a diameter of $1.2 \mathrm{~cm}$ and a length of $6 \mathrm{~cm}$, whereas for 
RT 42 and PX 42, a $11 \mathrm{~cm}^{3}$ thin-walled cylindrical cell with a diameter of $1.6 \mathrm{~cm}$ and a length of $7 \mathrm{~cm}$ was used. Both cells were made of tantalum for low heat capacity, good thermal conductance, and chemical inertness. On the outside of both cells, an electric heating wire was distributed and glued over the entire length. For the temperature measurements, small sensitive thermistors are incorporated in the cell wall.

Adiabatic scanning calorimetry (ASC) measurements results are also reported for the pure salt hydrate calcium chloride hexahydrate $\left(\mathrm{CaCl}_{2} \cdot 6 \mathrm{H}_{2} \mathrm{O}\right)$. The product was purchased from Sigma-Aldrich $\mathrm{GmbH}$, Switzerland, and had a quoted purity of $99.0 \%$, and a quoted melting temperature of $28^{\circ} \mathrm{C}$. This compound was measured in the $5 \mathrm{~cm}^{3}$ sample cell.

\section{Results and Discussion}

In Table 1 and in Figs. 3, 4, 5, 6, 7, an overview of the results obtained for the three paraffin-based PCMs, obtained from Rubitherm $\mathrm{GmbH}$, and for the pure PCM compound calcium chloride hexahydrate is given. The characteristics of the ASC runs as well as relevant results for the different materials are included in Table 1. The characteristics of the ASC runs are: the temperature ranges covered, the measuring times of each run, and the average heating or cooling rates of the runs. For the different PCMs the transition temperatures $T_{\text {tr }}$ are also given. For heating runs this is the temperature at which the whole sample has melted, and for cooling runs, the temperature where the sample starts to solidify. In both cases $T_{\text {tr }}$ is marked as sharp slope changes in the enthalpy curves. Also values for enthalpy changes $\Delta H$ over the transitions are given. For this quantity we have chosen the enthalpy differences between $T_{\operatorname{tr}}-5^{\circ} \mathrm{C}$ and $T_{\operatorname{tr}}+5^{\circ} \mathrm{C}$. Also values for the specific heat capacity, $C_{p}$, are included for the solid as well as for the liquid phase. For the solid phase this value corresponds with $T_{\mathrm{tr}}-10^{\circ} \mathrm{C}$, and for liquid phase with $T_{\operatorname{tr}}+5^{\circ} \mathrm{C}$.

Table 1 Characteristics and results of different ASC runs

\begin{tabular}{lllll}
\hline & $\mathrm{RT} 27$ & $\mathrm{RT} 42$ & $\mathrm{PX} 42$ & $\mathrm{CaCl}_{2} \cdot 6 \mathrm{H}_{2} \mathrm{O}$ \\
\hline$T$-range heating $\left({ }^{\circ} \mathrm{C}\right)$ & $5-35$ & $34-51$ & $28-47$ & $5-35$ \\
$T$-range cooling $\left({ }^{\circ} \mathrm{C}\right)$ & $35-5$ & $51-29$ & $47-29$ & $35-5$ \\
Measurement time heating (h) & 248 & 68 & 10 & 140 \\
Measurement time cooling $(\mathrm{h})$ & 176 & 380 & 137 & 128 \\
Average heating rate $\left(\mathrm{K} \cdot \mathrm{h}^{-1}\right)$ & 0.12 & 0.25 & 1.9 & 0.22 \\
Average cooling rate $\left(\mathrm{K} \cdot \mathrm{h}^{-1}\right)$ & 0.17 & 0.06 & 0.13 & 0.23 \\
$T_{\mathrm{tr}}$ heating $\left({ }^{\circ} \mathrm{C}\right)$ & 27.3 & 42.2 & 44.5 & 31.0 \\
$T_{\mathrm{tr}}$ cooling $\left({ }^{\circ} \mathrm{C}\right)$ & 27.2 & 41.0 & 43.7 & 30.3 \\
$\Delta h$ heating between $T_{\mathrm{tr}} \pm 5 \mathrm{~K}\left(\mathrm{~kJ} \cdot \mathrm{kg}^{-1}\right)$ & 165 & 138 & 62 & 178 \\
$\Delta h$ cooling between $T_{\mathrm{tr}} \pm 5 \mathrm{~K}\left(\mathrm{~kJ} \cdot \mathrm{kg}^{-1}\right)$ & 169 & 126 & 51 & 171 \\
$C_{p}$ solid at $T_{\mathrm{tr}}-10 \mathrm{~K}\left(\mathrm{~kJ} \cdot \mathrm{kg}^{-1} \cdot \mathrm{K}^{-1}\right)$ & 3.7 & 3.5 & 2.7 & 2.8 \\
$C_{p}$ liquid at $T_{\mathrm{tr}}+5 \mathrm{~K}\left(\mathrm{~kJ} \cdot \mathrm{kg}^{-1} \cdot \mathrm{K}^{-1}\right)$ & 2.6 & 2.3 & 1.9 & 2.4 \\
\hline
\end{tabular}





Fig. 3 Lower part: temperature dependence of the enthalpy of the PCM compound RT 27, the thick curve is a heating run, the thin one a cooling run. Upper part: corresponding to effective specific heat capacity results

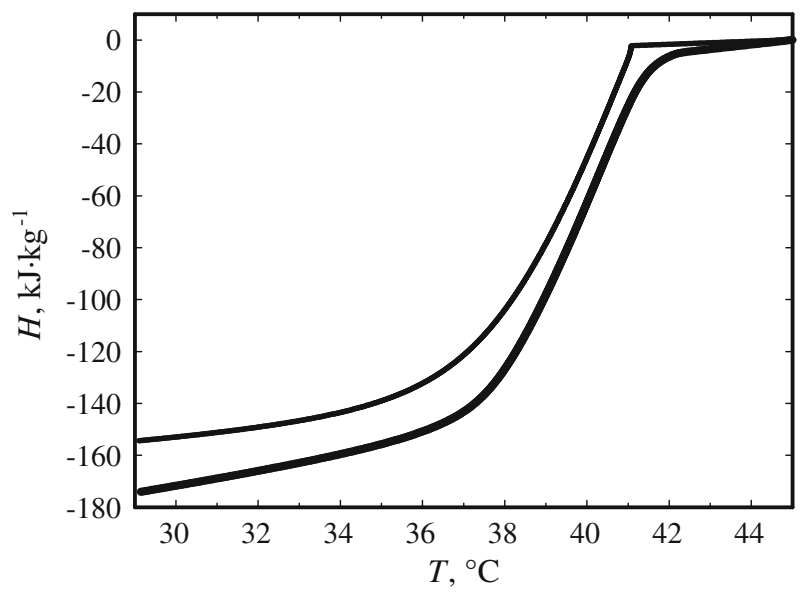

Fig. 4 Enthalpy as a function of temperature for the Rubitherm PCM RT 42. The thick curve is a heating run and the thin curve a cooling run 


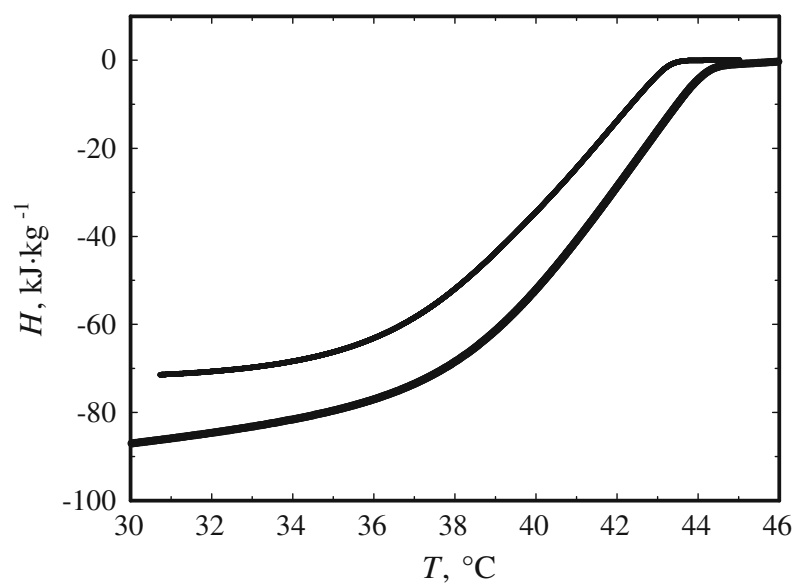

Fig. 5 Enthalpy as a function of temperature for the Rubitherm PCM PX 42. The thick curve is a heating run and the thin curve a cooling run

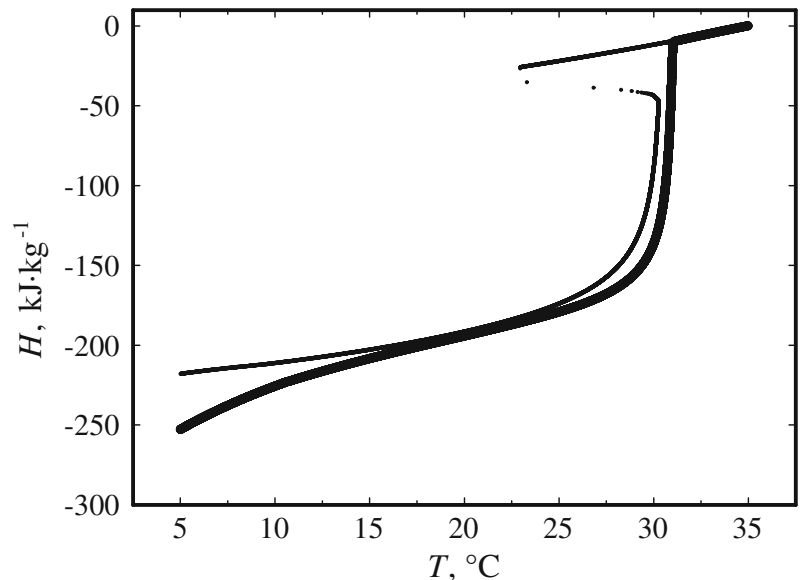

Fig. 6 Enthalpy as a function of temperature for calcium chloride hexahydrate. The thick curve is a heating run and the thin curve a cooling run

\subsection{Results for Paraffin-Based PCMs}

In Fig. 3, an overview of the results for the PCM RT 27 is given. In the lower part of that figure the temperature dependence of the enthalpy for a heating run is given by the thick curve and by the thin one for a cooling run. The top part of Fig. 3 represents the (effective) specific heat capacity, i.e., the derivative of the enthalpy curve. Here also the thick curve is for the heating run and the thin one is for the cooling run. It should be noted that in this (and the other figures) these curves are not fit curves through the data points but collections of very large numbers of closely spaced direct data points. From this figure it can be seen that there is not much difference in the enthalpy behavior and in the heat capacity behavior between the heating and cooling runs. 


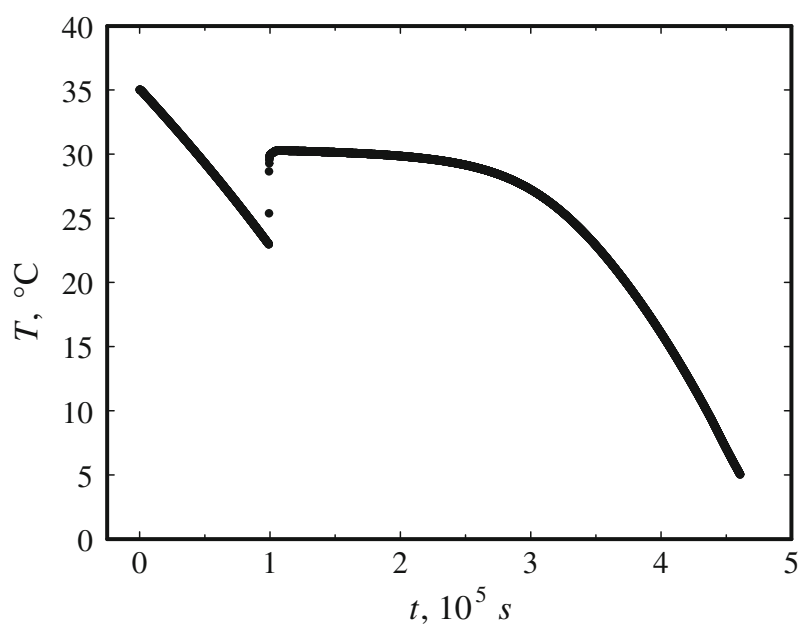

Fig. 7 Time dependence of the temperature during the cooling run on calcium chloride hexahydrate

In Fig. 4, the temperature dependence of the enthalpy is given for a heating (thick curve) and a cooling (thin curve) run in the RT 42 material. In Fig. 5 a similar plot is given for PX 42, which is a PCM material incorporated within a hydrophilic silica powder supporting structure. In both these samples (in particular for PX 42) there is a larger difference between the transition temperatures for heating and cooling. This signals most likely undercooling in the cooling runs. It is unlikely that the differences between heating and cooling runs are the result of temperature gradients over the sample. Calculations of the possible temperature difference, $\Delta T$, between the outside wall (with the heater) of the cylindrical cells and the center of the cell result, for all runs, in $\Delta T$ values of around $0.010 \mathrm{~K}$ or smaller, except for the fast heating run (of $1.9 \mathrm{~K} \cdot \mathrm{h}^{-1}$ ) on PX 42 where it was $\Delta T=0.16 \mathrm{~K}$. Thus, one has to conclude that some thermodynamic metastability must be at the origin of the subcooling. Moreover, it is possible that, in the grains (of $0.25 \mathrm{~mm}$ ), part of the PCM was not fully solidified.

The transition temperatures for RT 27 and RT 42 are in good agreement with the quoted ranges $\left(25^{\circ} \mathrm{C}\right.$ to $28^{\circ} \mathrm{C}$ for RT 27 and $37^{\circ} \mathrm{C}$ to $43^{\circ} \mathrm{C}$ for RT 42) by the manufacturer. For PX 42 our transition temperatures $\left(44.5^{\circ} \mathrm{C}\right.$ for heating and $43.7^{\circ} \mathrm{C}$ for cooling) are at the upper edge of the $38^{\circ} \mathrm{C}$ to $43^{\circ} \mathrm{C}$ range of the manufacturer. For the transition heat of RT 27 , a value of $184 \mathrm{~kJ} \cdot \mathrm{kg}^{-1}$ (no temperature range indicated) is given by Rubitherm, this is comparable to, but somewhat larger than our heating run value of $165 \mathrm{~kJ} \cdot \mathrm{kg}^{-1}$ for $\Delta H$ between $T_{\mathrm{tr}}-5^{\circ} \mathrm{C}$ and $T_{\mathrm{tr}}+5^{\circ} \mathrm{C}$. Most likely a larger $T$-range has been considered. The Rubitherm values for the heat of transition of $174 \mathrm{~kJ} \cdot \mathrm{kg}^{-1}$ (between $35^{\circ} \mathrm{C}$ and $50^{\circ} \mathrm{C}$ ) for RT 42 and of $114 \mathrm{~kJ} \cdot \mathrm{kg}^{-1}$ for PX 42 are much larger than our values in Table 1 over a narrower temperature range. Over the same $35^{\circ} \mathrm{C}$ to $50{ }^{\circ} \mathrm{C}$ range we obtained $160 \mathrm{~kJ} \cdot \mathrm{kg}^{-1}$ and $80 \mathrm{~kJ} \cdot \mathrm{kg}^{-1}$ for RT 42 and PX 42, respectively. For the specific heat capacity the supplier quotes the values $1.8 / 2.4 \mathrm{~kJ} \cdot \mathrm{kg}^{-1} \cdot \mathrm{K}^{-1}$ for RT 27 and RT 42 and a value of $1.6 \mathrm{~kJ} \cdot \mathrm{kg}^{-1} \cdot \mathrm{K}^{-1}$ for $\mathrm{PX}$ 42. The larger values are in reasonable agreement with our values in Table 1 for the liquid phase. Our solid-phase values at $T=T_{\mathrm{tr}}-10^{\circ} \mathrm{C}$ are much larger and are 
still subject to pretransitional heat-capacity increases. True solid-phase values should thus be obtained much deeper in the solid phase.

In spite of the strongly first-order character of these transitions, the $H(T)$ and $C_{p}(T)$ curves exhibit quite some pretransitional temperature variation on the low-temperature side of the transition, in such a way that it makes determining the beginning of the two-phase range on the low-temperature side difficult. Similar pretransitional effects in the vicinity of strongly first-order melting transitions have been observed in highresolution calorimetric studies of liquid crystals $[5,6,11]$. These effects are ascribed to premelting of hydrocarbon tails in the crystalline phase with increasing temperature, and gradual loss of the orientational order around the chain axes [11]. In the present case similar effects might occur in the paraffin hydrocarbon chains.

\subsection{Results for Calcium Chloride Hexahydrate}

In Fig. 6, the temperature dependence of the enthalpy of the PCM calcium chloride hexahydrate $\left(\mathrm{CaCl}_{2} \cdot 6 \mathrm{H}_{2} \mathrm{O}\right)$ for a heating (thick curve) as well as for a cooling (thin curve) run is given. The unusual shape of the curve for the cooling run at the hightemperature side is the result of substantial supercooling and then a sudden release of solidification heat in the thermally well insulated cell, resulting in an abrupt increase of the temperature to the real melting temperature of the substance. This can clearly be seen in Fig. 7 where the cell temperature is plotted as a function of time. Supercooling occurred to about $23^{\circ} \mathrm{C}$. At that temperature the sample suddenly solidified and the temperature raised to near the melting temperature. Further enthalpy release (to the controlled thermal environment of the cell) occurred in the continuation of the cooling run. In the heating run and also in the cooling run, pretransitional enthalpy changes are clearly present, similar to what is observed for the paraffin-based PCMs. However, the argument of increased chain mobility does not apply here. Apparently, as pointed out by one of the reviewers, this PCM melts incongruently due to a peritectic transition [1].

\section{Summary and Conclusions}

In this paper, ASC is introduced as a suitable tool for simultaneous measurements of the temperature dependence of the enthalpy and of the (effective) heat capacity of PCMs near their solid-liquid phase transition. Measurements have been performed at very slow heating and cooling rates, typically three orders of magnitude slower than the ones usually applied in differential scanning calorimetry (DSC). Moreover, because of the very slow rates in heating the samples, the ASC measurements result in the equilibrium temperature dependence of the enthalpy. Also in slow cooling runs, undercooling can potentially be eliminated or strongly reduced, depending on the sample type. Results are reported for three paraffin- and wax-based PCMs obtained from an industrial supplier (Rubitherm $\mathrm{GmbH}$, Germany). Results are also presented for the pure PCM salt hydrate material calcium chloride hexahydrate $\left(\mathrm{CaCl}_{2} \cdot 6 \mathrm{H}_{2} \mathrm{O}\right)$. An overview of characteristics of the ASC runs as well as values of relevant quantities obtained for the four samples are given in Table 1. Results are also represented in several figures. As a consequence of the very slow scanning rate, small differences (less 
than $0.1 \mathrm{~K}$ ) are seen in the transition temperatures for the paraffin-based PCMs between heating and cooling. On the high-temperature (liquid) side of the transitions, the slope discontinuity of the enthalpy curve is quite sharp, while on the low-temperature (solid) side, the changes are more gradual and rounded. For the heating run in the salt hydrate the shape of the enthalpy curve is analogous to that of the paraffin-based materials. However, for the cooling run in the salt hydrate substantial undercooling (of about $7^{\circ} \mathrm{C}$ ) was observed.

Acknowledgments The authors thank the Research Foundation-Flanders (FWO) for financial support (project G.0230.07). J.L. acknowledges FWO for a postdoctoral fellowship.

\section{References}

1. H. Mehling, L.F. Cabeza, Heat and Cold Storage with PCM-An up to Date Introduction into Basics and Applications (Springer, Berlin, Heidelberg, 2008)

2. Z. Liu, D.D.L. Chung, Thermochim. Acta 366, 135 (2001), and references therein

3. A. Genovese, G. Amarasinghe, M. Glewis, D. Mainwaring, R.A. Shanks, Thermochim. Acta 443, 235 (2006), and references therein

4. E. Günther, S. Hiebler, H. Mehling, R. Redlich, Int. J. Thermophys. 30, 1257 (2009)

5. J. Thoen, H. Marynissen, W. Van Dael, Phys. Rev. A 26, 2886 (1982)

6. G. Cordoyiannis, L.F.V. Pinto, M.H. Godinho, C. Glorieux, J. Thoen, Phase Transitions 83, 280 (2009)

7. J. Thoen, G. Cordoyiannis, C. Glorieux, Liquid Cryst. 36, 669 (2009)

8. J. Thoen, High resolution adiabatic scanning calorimetry and heat capacities. in Heat Capacities: Liquids, Solutions and Vapours, chap. 13, ed. by E. Wilhelm, T. Letcher (The Royal Society of Chemistry, London, 2010), pp. 287-306

9. G. Pitsi, J. Caerels, J. Thoen, Phys. Rev. B 55, 915 (1997)

10. B. Van Roie, J. Leys, K. Denolf, C. Glorieux, G. Pitsi, J. Thoen, Phys. Rev. E 72, 041702 (2005)

11. K.J. Lushington, G.B. Kasting, C.W. Garland, J. Phys. Lett. 41, L419 (1980) 